\title{
HYPERPLANE SECTIONS OF LINEARLY NORMAL CURVES
}

\author{
E. BALLICO
}

(Communicated by Eric Friedlander)

\begin{abstract}
A particular case of the results proved here is that a general set of $d$ points in $\mathbf{P}^{r-1}(k)$ is not the hyperplane section of a linearly normal, locally complete intersection curve in $\mathbf{P}^{r}(k)$ if $r \geq 6, d \geq \max (15, r+3)$, and the characteristic of $k$ is zero.
\end{abstract}

In the last few years the following types of problems have been considered by several authors (see [CO, BM, B, W1, W2]): fix a hyperplane $H$ of $\mathbf{P}^{r}$, and take a finite subset $A$ of $H(\operatorname{card}(A)=d)$; under what conditions on $A$ and $X$ can we find a curve $X \subset \mathbf{P}^{r}$ of degree $d$ such that $X \cap H=A$ ? A natural assumption is that this should hold (for a given class of curves) when $A$ is a general set of $d$ points of $H$. A natural assumption on $X$ is that $X$ is arithmetically Cohen-Macaulay and locally a complete intersection. Under these assumptions the answer is affirmative if $r=3$ [CO], while, in any characteristic, the answer is often negative if $r \geq 6$ [B]. Under these assumptions on $A$ and $X$ (and characteristic 0 ) the picture was completely described in the recent paper [W1]. The first aim of this note is to show how the ideas contained in [W1] give a simple proof of the following striking results.

Theorem 0.1. Assume characteristic 0 . Fix integers $d, p_{a}, r$ with any one of the following.

$$
\begin{aligned}
& r \geq 12 \text { and } d \geq r+3, \\
& r=11 \text { and } d \geq 15, \\
& r=10 \text { and } d \geq 14, \\
& 7 \leq r \leq 9 \text { and } d \geq 13, \\
& r=6 \text { and } d \geq 15 .
\end{aligned}
$$

Let $M$ be any irreducible component of $\mathrm{Hilb}\left(\mathbf{P}^{r}\right)_{\text {red }}$ whose general member corresponds to a locally complete intersection curve $X$ of degree $d$ and arithmetic genus $p_{a}$ which is also linearly normal. Fix a hyperplane $H$ of $\mathbf{P}^{r}$. Then a general set of $d$ points of $H$ is not the hyperplane section of a curve in $M$.

Theorem 0.1 is just a particular case (the case $k=1$ ) of the following result.

Received by the editors January 27, 1993.

1991 Mathematics Subject Classification. Primary 14H99, 14 N05.

Key words and phrases. Projective curve, Hilbert scheme, locally complete intersection, deformation functor, normal sheaf.

The author was partially supported by MURST and GNSAGA of CNR (Italy). 
Theorem 0.2. Assume characteristic 0. Fix integers $d, p_{a}, r, k$ with $r \geq 4$, $k \geq 2, d \geq r$, and

$$
(2 d-4) /(r-3)>k d+1-p_{a}-\left[\left(r_{k}\right) ! /(r ! k !)\right] .
$$

Let $M$ be any irreducible component $\mathrm{Hilb}\left(\mathbf{P}^{r}\right)_{\text {red }}$ whose general member corresponds to a locally complete intersection curve $X$ of degree $d$ and arithmetic genus $p_{a}$ for which the restriction map $\rho(k): H^{0}\left(\mathbf{P}^{r}, \mathbf{O}(k)\right) \rightarrow H^{0}\left(X, \mathbf{O}_{X}(k)\right)$ is surjective. Fix a hyperplane $H$ of $\mathbf{P}^{r}$. Then a general set of $d$ points of $H$ is not hyperplane section of a curve in $M$.

Note that if $k=1$, the inequality (1) is satisfied exactly for the pairs $(d, r)$ considered in the statement of Theorem 0.1 . If $k=2$, Theorem 0.2 works for $r=4$ when $d \geq 31$ and for $r=5$ when $d \geq 19$.

In Theorem 0.1 "linearly normal" means that the curve $X$ (which spans $\mathbf{P}^{r}$ since its hyperplane section spans $\left.\mathbf{P}^{r-1}\right)$ has $h^{0}\left(X, \mathbf{O}_{X}(1)\right)=r+1$; if $X$ is integral this does not mean that the corresponding linear system induced on the normalization, $C$, of $X$ is complete. From the point of view of the abstract curve $C$ it is more important to consider all its birational morphisms into $\mathbf{P}^{r}$ with image of degree $d$, even if the images have different arithmetic genera. This is exactly (varying $C$ ) the point of view of Chow varieties or, better, of that small part of the Chow scheme of degree $d$ one-dimensional cycles on $\mathbf{P}^{r}$ parametrizing integral curves whose normalization has fixed genus. Furthermore, from this point of view the "locally complete intersection" assumption on $X$ (which comes from the essential use of [W1, Theorem 5]) is very restrictive; for instance, if $X$ has a point with $m$ ordinary branches with linearly independent tangents and $m \geq 3$, then $X$ is not locally a complete intersection at $P$. For this reason (and from the point of view of the Chow scheme) the following result is interesting.

Theorem 0.3. Assume characteristic 0. Fix integers $d, g, r, k$ with $r \geq 4$, $k \geq 2, d \geq r$, and

$$
(2 d-4) /(r-3)<k d+1-g-[(r+k) ! /(r ! k !)] .
$$

Fix a hyperplane $H$ of $\mathbf{P}^{r}$. Then a general set of $d$ points of $H$ is not the hyperplane section of the image, $X$, of a birational morphism $\pi: C \rightarrow X$ with $C$ a smooth curve of genus $g, \operatorname{deg}(X)=d$, and such that, setting $L:=\pi^{*}\left(\mathbf{O}_{X}(1)\right)$, the induced restriction map $\rho(k): \pi^{*}\left(H^{0}\left(\mathbf{P}^{r}, \mathbf{O}(k)\right)\right) \rightarrow H^{0}\left(C, L^{\otimes k}\right)$ is surjective.

\section{THE PROOFS}

Note again that Theorem 0.1 is just the case " $k=1$ " of Theorem 0.2 . Here is a short proof of Theorem 0.2 .

Proof of Theorem 0.2. By [W1, Theorem 5] we have $p_{a}(X) \leq(2 d-4) /(r-3)$. Since by assumption we have $h^{0}\left(X, \mathbf{O}_{X}(k)\right) \leq[(r+k) ! /(r ! k !)]$, by RiemannRoch we have $p_{a}(X) \geq d+1-[(r+k) ! /(r ! k !)]$, contradicting inequality (2).

Remark 1.1. By increasing $r$ (in Theorem 0.1) we can weaken the hypothesis on linear normality, e.g., if we assume that $h^{0}\left(X, \mathbf{O}_{X}(1)\right) \leq r+1+\alpha$ and have $(r-3)(d-r-\alpha)<(2 d-4)$ (e.g., we may take $r \geq 6$ and $d \geq 16+3 \alpha)$. 
It is known (see [BM] and [W2] for much more) that a general subset of $H$ is the hyperplane section of a smooth rational curve (not linearly normal if $d>r$ ). Sometimes it is also the hyperplane section of a singular curve, $X$, whose normalization is $\mathbf{P}^{1}$. But by Theorem 0.1 the singularities of $X$ are restricted by the fact that $h^{0}\left(X, \mathbf{O}_{X}(1)\right)=r+1$ (or much lower than $d+1$ by Remark 1.1).

Proof of Theorem 0.3. As in the proof of Theorem 0.2, it will be sufficient to obtain in our different situation the conclusion of [W1, Theorem 5], i.e., the inequality $(r-3) g \leq(2 d-4)$. To check this we will use a result of $[\mathrm{AC}, \S 6]$, and several ideas/tricks contained in the proof of [W1, Theorem 5]. Let $N_{\pi}$ be the conormal sheaf to $\pi$; by Horikawa's definition (see [Ho] or [AC, §5]) $N_{\pi}$ can be defined as the cokernel of the map $T C \rightarrow \pi^{*}\left(T \mathbf{P}^{r}\right)$ induced by $\pi$. Let $K$ be the torsion part of $N_{\pi}$; set $N^{\prime \prime}:=N_{\pi} / K$. Since $C$ is a smooth curve, the sheaf " $N$ " is locally free and of rank $r$. Indeed, we have $N_{\pi} \cong N^{\prime \prime} \oplus K$ because the appropriate Tor vanishes (use that $C$ is smooth and of dimension 1). Hence we have the following exact sequence on $C$ :

$$
0 \rightarrow T C \rightarrow \pi^{*}\left(T \mathbf{P}^{r}\right) \rightarrow K \oplus N^{\prime \prime} \rightarrow 0 \text {. }
$$

Since $X \cap H$ is reduced, $\operatorname{Hilb}^{d}(H)$ is smooth at $X \cap H$ and of dimension $d(r-1)$. Let $Y$ be the tangent space of $\operatorname{Hilb}^{d}(H)$ at $X \cap H$. We may assume that the morphism $\pi$ defines a general point of the corresponding part of the "Chow variety" (the part parametrizing integral curves with normalization of genus $g$ ). By the assumption of Theorem 0.3 the corresponding map from pairs $(C, \pi)$ to $\mathrm{Hilb}^{d}(X)$ obtained upon taking intersection with $H$ dominates the component of $\operatorname{Hilb}^{d}(X)$ containing the reduced schemes of length $d$. Since we are in characteristic 0 and we are interested in the general set of $d$ points of the hyperplane $H$ and in the general pair $(C, \pi)$, we may assume (changing $(C, \pi)$ ) that the linear map $u: H^{0}\left(C, N_{\pi}\right) \rightarrow Y$ of tangent spaces to the corresponding deformation functors is surjective. Since we may assume that $(C, \pi)$ is general, nearby deformations of $\pi$ (moving also $C$ ) are equisingular. Hence by $[\mathrm{AC}, \S 6]$, we see that $u(K)=0$. Hence the induced linear map $v: H^{0}\left(N^{\prime \prime}\right) \rightarrow Y$ is surjective. Note that we may identify $Y$ with $H^{0}\left(\mathbf{O}_{Z}\right)$, where $Z$ is the zero locus of the section of $L$ induced by the section, $s$, of $H^{0}\left(\mathbf{P}^{r}, \mathbf{O}(1)\right)$ giving the equation of $H$ (i.e., $Z$ are the $d$ points with $\pi(Z)=X \cap H)$. Since $\pi$ is smooth at each point of $Z$, the section $s$ induces the following exact sequences:

$$
\begin{aligned}
& 0 \rightarrow N_{\pi} \otimes L^{*} \rightarrow N_{\pi} \rightarrow N_{\pi} \mid Z \rightarrow 0 \\
& H^{0}\left(C, N_{\pi} \otimes L^{*}\right) \rightarrow H^{0}\left(C, N_{\pi}\right) \rightarrow H^{0}\left(N_{\pi} \mid Z\right) \\
& \rightarrow H^{1}\left(C, N_{\pi} \otimes L^{*}\right) \rightarrow H^{1}\left(C, N_{\pi}\right) .
\end{aligned}
$$

The identification just made of the tangent spaces to the corresponding deformation functors gives the surjectivity of the second map in (5), hence the injectivity of the last map in (5). Since $K$ has finite support, we have $H^{1}\left(C, N_{\pi} \otimes L^{*}\right)=$ $H^{1}\left(C, N^{\prime \prime} \otimes L^{*}\right)$ and $H^{1}\left(C, N_{\pi}\right)=H^{1}\left(C, N^{\prime \prime}\right)$. Hence we have the injectivity of the induced map $H^{1}\left(C, N^{\prime \prime} \otimes L^{*}\right) \rightarrow H^{1}\left(C, N^{\prime \prime}\right)$. Since $N^{\prime \prime}$ is locally free, the last part of the proof of [W1, Theorem 5] gives (verbatim!) that 
$H^{1}\left(C, N^{\prime \prime}\right)=0$. Since $\operatorname{deg}\left(N^{\prime \prime}\right) \leq \operatorname{deg}\left(N_{\pi}\right)=(r+1) d+2 g-2$ by $(3)$ and $N^{\prime \prime}$ is locally free of rank $r-1$, while $\operatorname{dim}(Y)=(r-1) d$, by Riemann-Roch we obtain $(r-3)(g-1) \leq 2 d$, as wanted.

\section{ACKNOWLEDGMENTS}

Obviously this paper is a byproduct of [W1]; I want to thank C. Walter very much for sending me his preprint. J. Migliore first interested me in these kinds of problems, and to him I would like to say, "Thanks a lot".

\section{REFERENCES}

[AC] E. Arbarello and M. Cornalba, Su una congettura di Petri, Comment. Math. Helv. 56 (1981), 1-38.

[B] E. Ballico, Points not as hyperplane sections of projectively normal curves, Proc. Amer. Math. Soc. 112 (1991), 343-346.

[BM] E. Ballico and J. Migliore, Smooth curves whose hyperplane section is a given set of points, Comm. Algebra 18 (1990), 3015-3040.

[CO] L. Chiantini and F. Orecchia, Plane sections of arithmetically normal curves in $\mathbf{P}^{3}$, Algebraic Curves and Projective Geometry (Trento 1988), Lecture Notes Math., vol. 1389, Springer-Verlag, New York, 1989, pp. 32-42.

[EH] D. Eisenbud and J. Harris, The dimension of the Chow variety of curves, Compositio Math. 83 (1992), 291-310.

[Ho] E. Horikawa, On deformations of holomorphic maps. I, J. Math. Soc. Japan 25 (1973), 372-396.

[W1] C. Walter, Hyperplane sections of arithmetically Cohen-Macaulay curves, preprint.

[W2] _ Hyperplane sections of curves of small genus, preprint.

Department of Mathematics, University of Trento, 38050 Provo, Trento, Italy

E-mail address: (bitnet) ballicoeitncisca or ballico@itnvax.science.unitn.it 